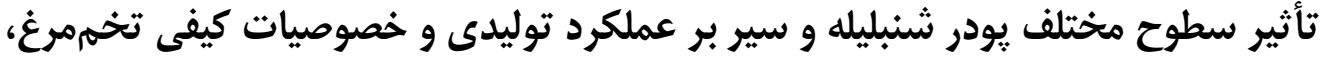

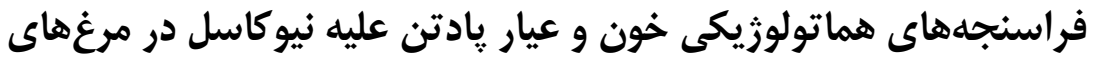



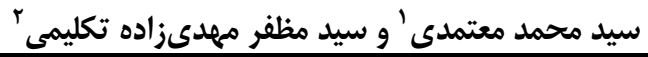

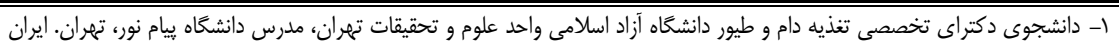

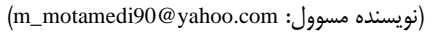 \\ r- عضو هيئت علمى مؤسسه تحقيقات علوم دامى كشور، كرج. ايران

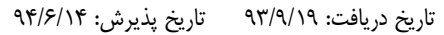



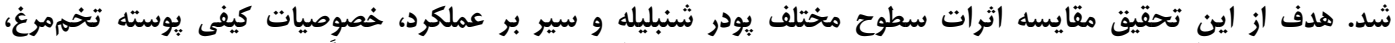

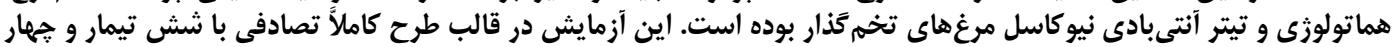

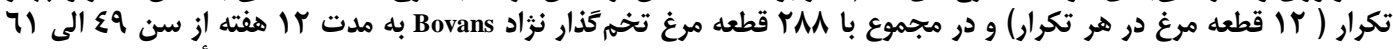

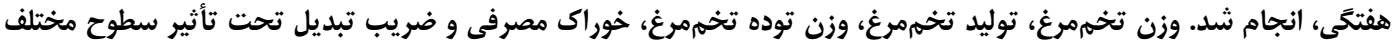

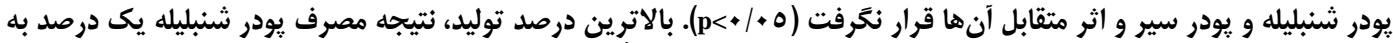

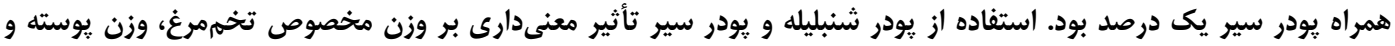



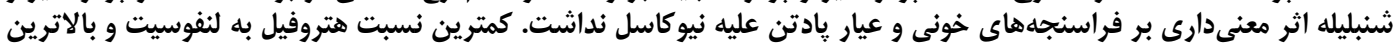

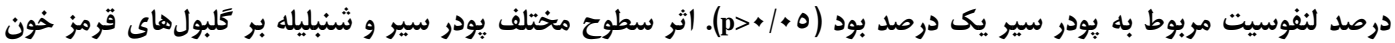



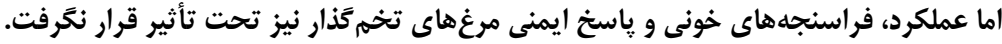

وازههاى كليدى: يودر سير، يودر شنبليله، عملكرد، عيار يادتن، فراسنجههاى خونى و مرغ تخمَذذار

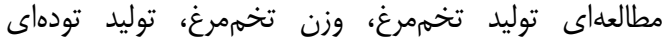



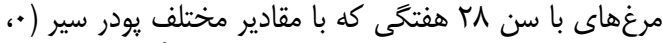

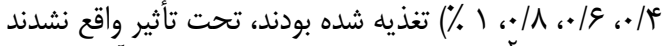

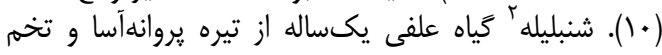

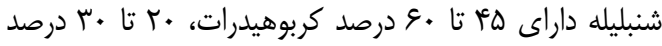

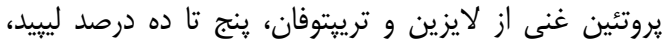

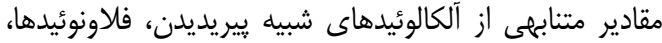

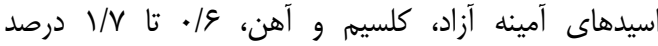


سيتوسترول، ويتامينها (A)، B

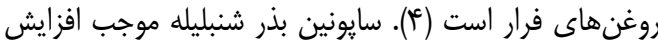

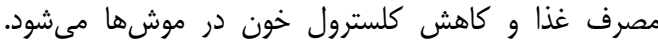

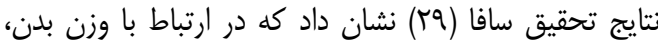



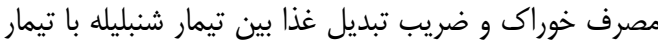

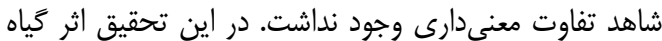

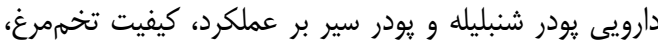


تخمثذار تجارى مورد بررسى قرار گرفتنه است.

1- Garlic (Allium sativum)
كياهان دارويى از جمله مواد افزودنى طبيعى هستند كه كأن

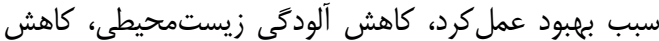

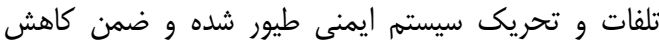

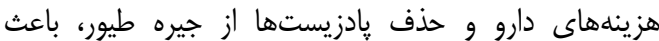

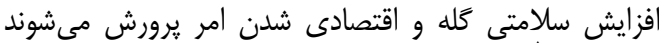

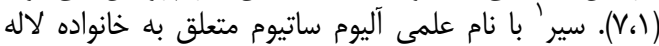



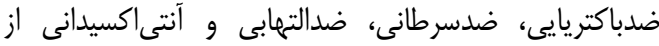

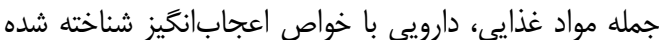

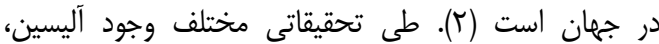

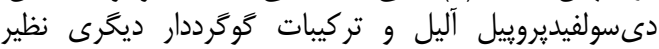

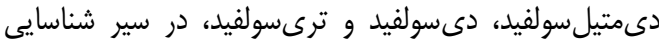



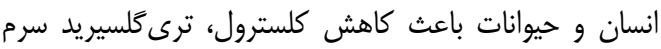

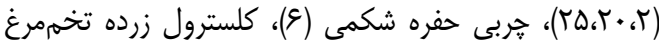

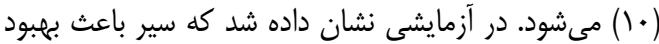

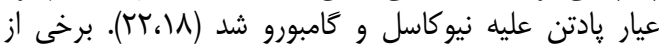



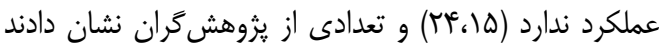

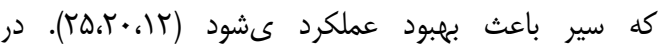

2- Fenugreek-Trigonella foenum-graecum (Papilionaceae) 










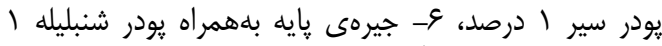

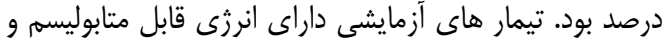

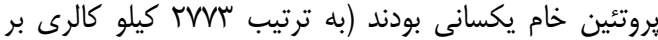

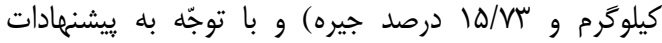

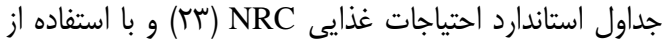

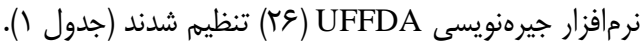

\section{مواد و روشها}

اين آزمايش در مؤسسه تحقيقات علوم دام دامى كشور واقع إن





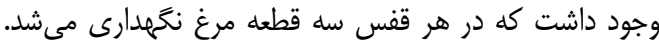

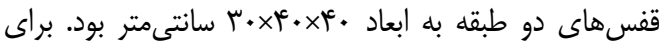

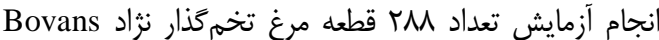

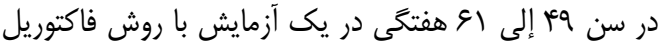

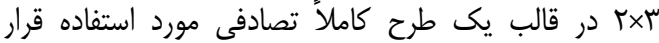

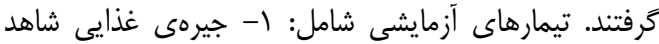

Table 1. The diet composition (components of diet in ration based hundred percent)

جدول ا- تركيب جيره غذايى (اجزاى جيره بر حسب درصد)

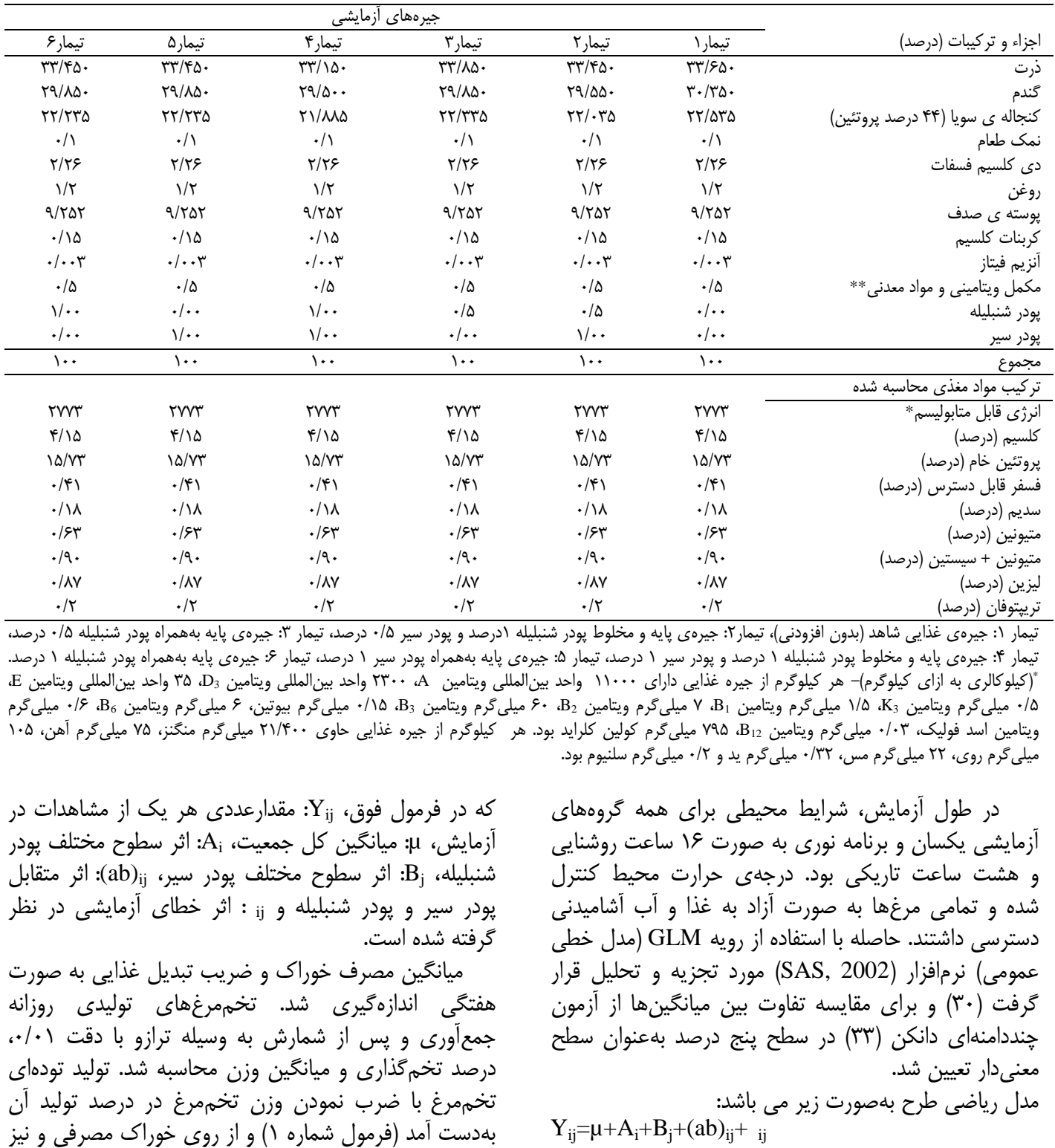




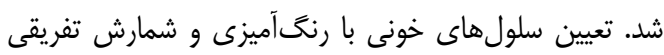

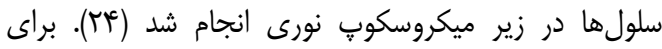

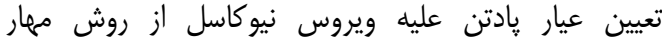
هماكلوتيناسيون ميكروتيتر (HI) استفاده شد (بآبار).

\section{نتايج و بحث}

همانطورى كه در جدول r مشاهده مى شود استفاده از

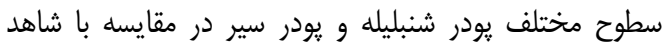

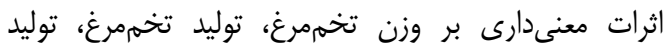



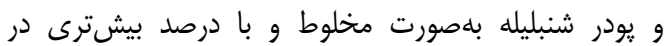

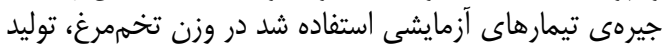

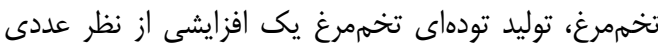



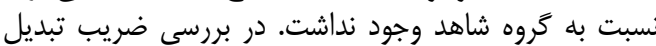







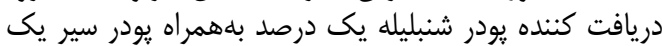

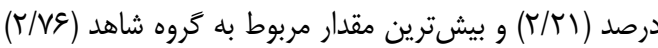


اختاف معنىدارى وجود نداشت ولى استفاده از سطوح يك تيك

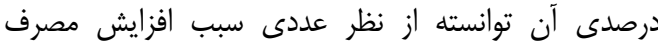

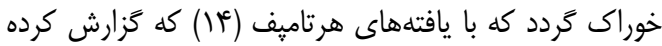

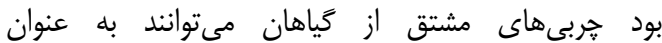





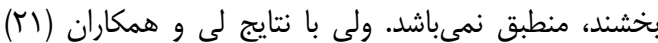



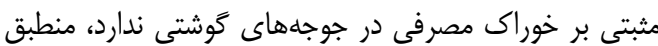

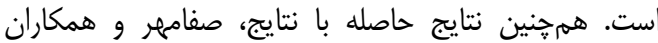



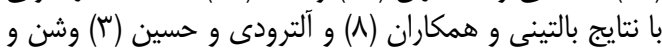



توليد تودهاى تخممرغ، ضريب تبديل خوراك براى هر يك از



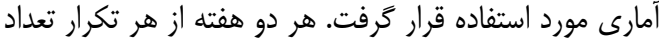

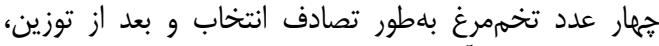

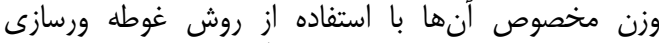

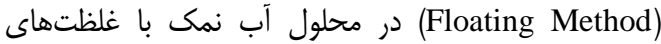



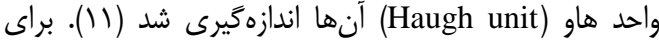
اندازمخيرى واحد هاو از فرمول (َّ) استفاده شده است.


توليد توده تخممرغ (كرم) (1).

توليد تودماى تخمصرغ (كراء (كرم)

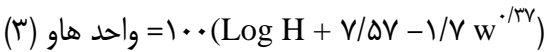

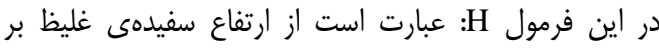

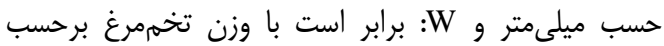

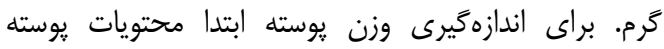



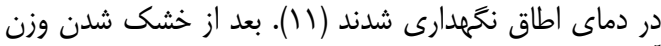

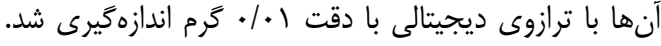

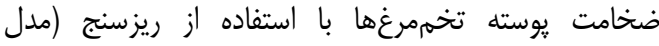

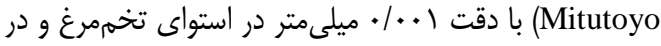



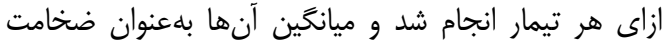





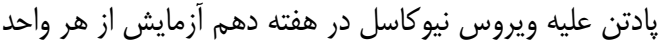



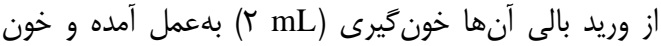

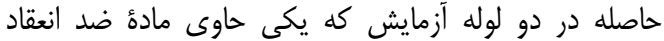



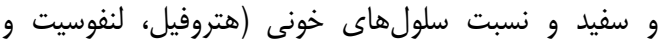

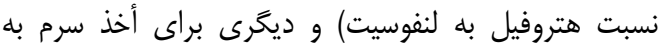



جدول r- اثريودر شنبليله و يودر سير بر عملكرد مرغان تخمكذار عايه Table 2. Effect of garlic and fenugreek powder on performance of laying hens

\begin{tabular}{|c|c|c|c|c|c|}
\hline ضريب تبديل غذايى & خوراك مصرفى (كرم) & توليد تودهاى (كرم) & توليد تخممرغ (درصد) & وزن تخممرغ (گرم) & تيمارها \\
\hline$T / V G$ & $11 \cdot 11$ & $f 1 / \cdot 1$ & SD/AT & אT/Tr & بدون كياه دارويى(شاهد) \\
\hline$T / r \mu$ & $111 / r$ & $\mathrm{eq} / \mathrm{AV}$ & VN/Q & r & $\mathrm{F}_{\cdot / \mathrm{a}}$ \\
\hline$r / D \mid$ & $111 / 1$ & $|\oplus / 1|$ & $V \pi / g r$ & gr/DH & $F_{1}$ \\
\hline$T / T V$ & $111 / 4$ & $F q / \Delta \Lambda$ & $v 9 / .9$ & r & $\mathrm{G}_{1}$ \\
\hline$r / 4 q$ & 11. & r & $99 / v 9$ & 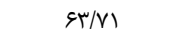 & $\cdot / \Delta+F_{1} G$ \\
\hline$r / T)$ & $111 / 8$ & $\Delta \cdot / 9$. & Vq/ar & gr/Ar & $1+F_{1} G$ \\
\hline$\cdot / \cdot v$ &.$/ 44$ & $1 / 49$ & $1 / 91$ & $\cdot|r|$ & SEM \\
\hline$\cdot / F^{c}$ & $\cdot / M$ &.$/ 19$ & . & $\cdot / \Delta$. & $P$-value \\
\hline
\end{tabular}


افزايش ترشح مواد هضم كنندهى مواد مغذّى از جمله آنزيمها

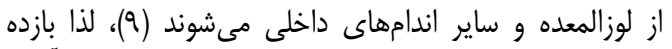

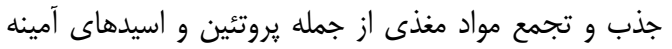

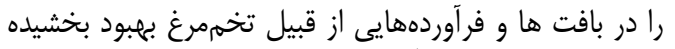

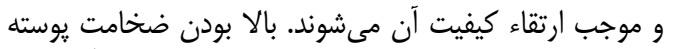

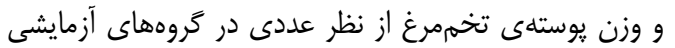

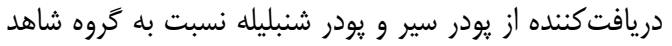



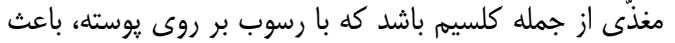

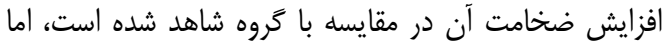

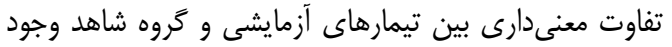

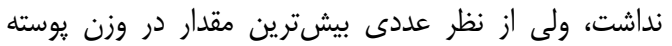

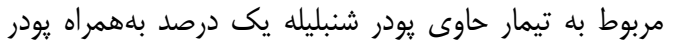

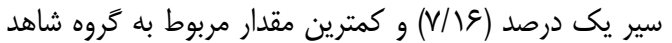

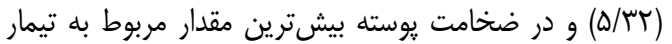



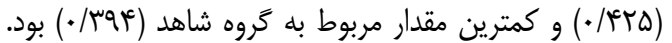

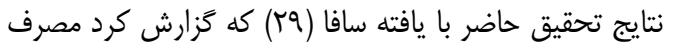



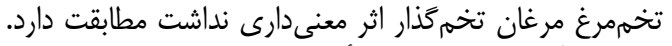

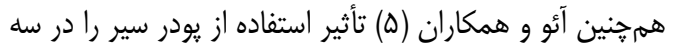







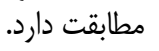

همانطورى كه در جدول r مشاهده مى شود استفاده از

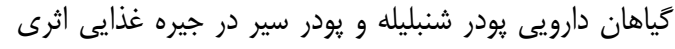

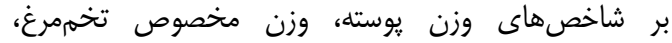

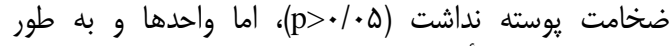

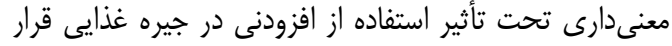

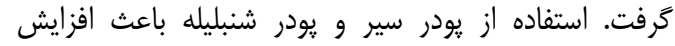

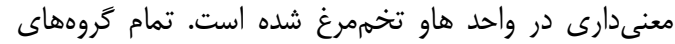

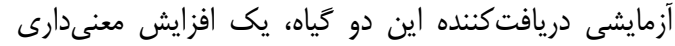

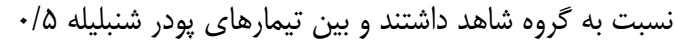



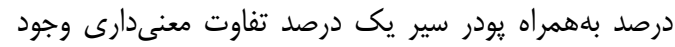



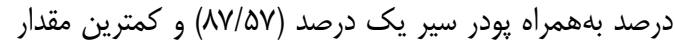



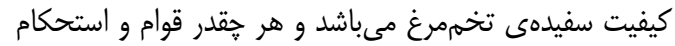

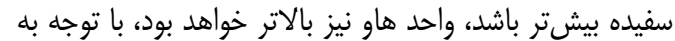

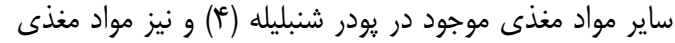
موجود در يودر سير شامل اسيدهاى آلى، اسيدهاى آميني، آمينه





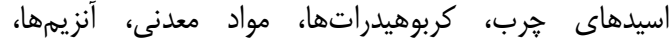

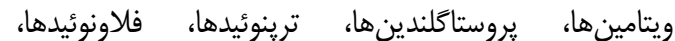

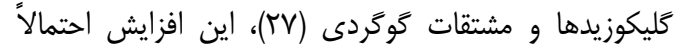

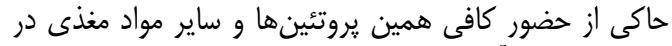

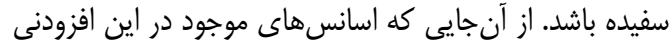



Table 3. Effect of garlic and fenugreek powder on egg quality of laying hens

\begin{tabular}{|c|c|c|c|c|}
\hline عدد هاو & وزن يوسته (كرم) & ضخامت يوسته & (ميلى كرم مخر ميلى ليتر مكعب) & تيمارها \\
\hline $\mathrm{c}_{V \varepsilon / \Lambda}$. & D/Tr &.$/$ raf & $1 / \cdot \wedge r$ & بدون گياه دارويى (شاهد) \\
\hline${ }^{\mathrm{ab}} \wedge \mathrm{r} / \Delta \wedge$ &  &.$/ 4 \cdot 1$ & $1 / . \wedge F$ & $\mathrm{~F}_{\mathrm{N} / \mathrm{\Delta}}$ \\
\hline${ }^{\mathrm{ab}} \wedge \mathrm{r} / \mathrm{Vr}$ & s/rq & $\cdot / 4 \cdot r$ & $1 / \cdot 11$ & $F_{1}$ \\
\hline${ }^{\mathrm{ab}} \wedge \mathrm{m} / \mathrm{qV}$ & 8/q &.$/ 41$. & $1 / 1 \mu$ & $\mathrm{G}_{1}$ \\
\hline${ }^{a} \Delta V / \Delta V$ & g/ץ &.$/ \% q$. & $1 / \cdot 1 \Delta$ & $\cdot / \Delta+F_{1} G$ \\
\hline${ }^{b} V q / T V$ & $\mathrm{~V} / \mathrm{l}$ & - IFTA & $1 / 18$ & $1+F_{1} G$ \\
\hline $1 . / 94$ &.$/ 14$ & $\cdot / \pi \Delta$ & $\cdot \cdots \Delta$ & SEM \\
\hline $.1 \cdot 0$ &.$/ 1 T$ & $\cdot / \cdot \mathrm{V}$ &.$/ 19$ & P-value \\
\hline
\end{tabular}



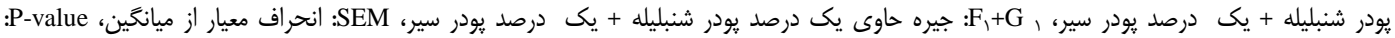
سطح معنى دارى.

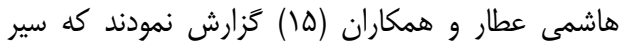

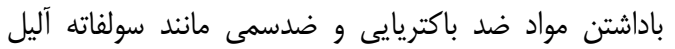

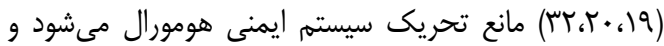

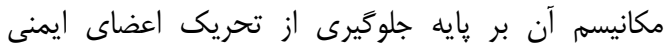



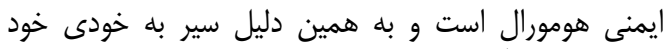




ايمنى بر عليه بيمارى نيوكاسل و كامبرو شد كه بان بانتايج اين
با توجه به جدول ع استفاده از اين افزودنىهاى كَّاهى

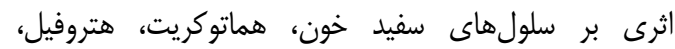



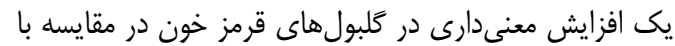

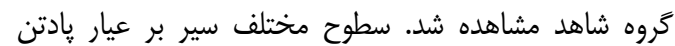
عليه ويروس نيوكاسل اثر معنى دارى نداشت. نتايج نشان


بى تأثير است و با كزارشهاى ساير محققين همخرانى دارد 
شده است. افزايش هتروفيلها اغلب نشانهى التهاب شناخته

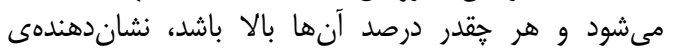

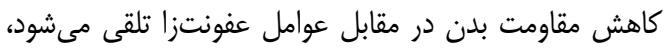





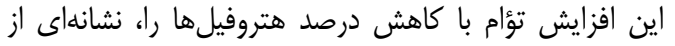

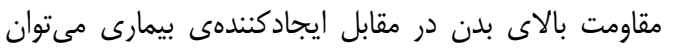

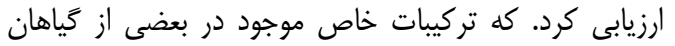


مهمى را در بهبود سيستم ايمنى مى توانند ايفا بكنند.
يثوهش منطبق نيست (عا). نتايج متناقص در آزمايشها با

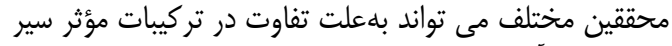

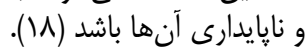

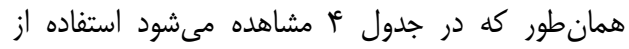

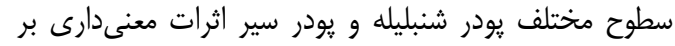

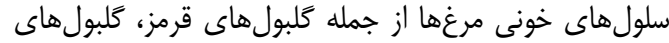

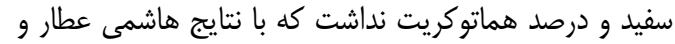

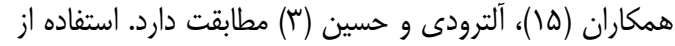

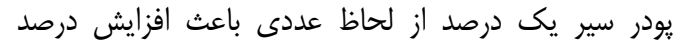
لنفوسيتها و نيز كاهش نسبت هتروفيلها به لن لنفوسيتها

جدول f- اثر يودر شنبليله و يودر سير بر فراسنجهاى خونى مرغان تخمكذار Table 4. Effect of garlic and fenugreek powder on blood parameters of laying hens

\begin{tabular}{|c|c|c|c|c|c|c|c|}
\hline عيار يادتن عليه & هتروفيل به & $\begin{array}{l}\text { لنفوسيت) } \\
\end{array}$ & هتروفيل) & (ميليون درميلى مترمكاى سفيد &  & 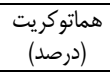 & تيمارها \\
\hline$V / \backslash Q$ & 每 & $9 V / V D$ & $4 \% / .9$ &  & $T / T f^{c c}$ & $r r / F q$ & بدون گياه دارويى (شاهد) \\
\hline $8 / 1 T$ & $\cdot / 4 \cdot 9$ & GN/IV & $r \cdot / V \Delta$ & $r t / I V$ & $T / \Lambda Q^{c}$ & Tו/IV & $\mathrm{F} \cdot / \Delta$ \\
\hline$V / T F$ & ./491 & SN/IF & $r \cdot / 4 \mid$ & سT/T & $r / r)^{\mathrm{ab}}$ & TM/Ar & $F_{1}$ \\
\hline$s / r \Lambda$ & س & $9 q / \ldots$ & $r \cdot / t r$ & tr/ta & $r / M \Lambda^{a}$ & Tr/Ta & $\mathrm{G}_{1}$ \\
\hline $9 / 4 \mid$ & • & SN/TD & $r \cdot / 4 q$ & $t r / \cdot \Delta$ & 年 & TI/qg & $\cdot / \Delta+F_{1} G$ \\
\hline$V / f \&$ & $\cdot / \mu \cdot 1$ & SN/NE & $r \cdot / g r$ & $4 T / 98$ & $T / T^{c b}$ & rt/T & $1+F_{1} G$ \\
\hline  & .1 .49 & $1 / K \Lambda$ & D/T & $\cdot / \Delta \wedge \varepsilon$ & $.1 \cdot 01$ & . & SEM \\
\hline.$/ 194$ & $\cdot 1 \cdot \mathrm{Vq}$ & $\cdot / \backslash \Delta S$ &.$/ 1 \cdot 9$ & -/VYS &.$/ \cdot T \Delta$ &.$/ 419$ & P-value \\
\hline
\end{tabular}

$$
\begin{aligned}
& \text { تشكر و قدردانى }
\end{aligned}
$$

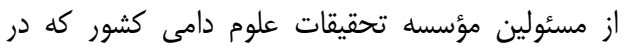

$$
\begin{aligned}
& \text { اجراى اين آزمايش ما را يارى نمودند تقدير و تشكر مى تشود. }
\end{aligned}
$$

$$
\begin{aligned}
& \text { استفاده از سطوح مختلف گياهان دارويى يودر شنبليله و }
\end{aligned}
$$

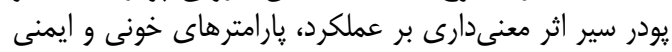

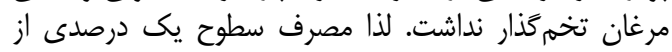

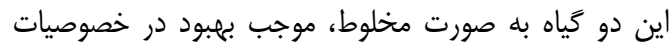

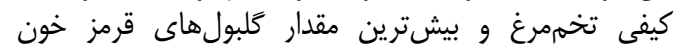

$$
\begin{aligned}
& \text { مرغهاى تخمثذار مى تهود. }
\end{aligned}
$$

منابع

1. Afshar Mazandaran, N. and A. Rajab. 2002. Probiotics and Their Use In Livestock And Poultries Feed. Second edition. Nourbakhsh Press, Tehran, 3: 141-152 (In Persian).

2. Aji, S.B., K. Ignatius, A. Adato, J.B. Nuho, U. Abdolkarim, A.U. Aliyu, M.B. Gombo, M.A. Ibrahim, I. Abubakar, M.M. Bukar, H.A. Imam and P.T. Numan. 2011. The effects of feeding onion (Alium cepa) and garlic (Alium sativum) some Performance Characteristics of broiler chickens. Research Journal of Poultry Science, 4: 22-27.

3. Al-Troudi, B. and A.K.S. Hussein. 2011. Effect of Supplementing fenugreek seeds powder on some productive and blood parameters of broiler chickens. Takrit University Magazine of Agriculturev Science, 4: 232-241.

4. American Botanical Council. 2000. Herbal Medicine: Expanded Commission E Monographs. Integrative Medicine Communications, Inc, 1: 241-249.

5. Ao, X., J.S. Yoo, J.H. Lee, H.D. Jang, J.P. Wang, T.X. Zhou and I.H. Kim. 2010. Effects of fermented garlic powder on production performance, egg quality, blood profiles and fatty acids composition of egg yolk in laying hens. Asian Australian Journal of Animal Science, 6: 786-791.

6. Ashryrizadeh, A., B. Daster and M. Shamsshargh. 2009. Use of garli, black cumin and wild mint in broiler chickens diets. Journal of Animal and Veterinary Advances, 8: 1860-1863.

7. Azarbaijani, A. and H. Jahanfar. 2001. Organic acids as growth stimulators in ruminant livestock. Journal of Livestock and Poultry, 37: 25-40 (In Persian).

8. Baltini, S., K. Ignatius, Y.A. Adatu Asha, J. Bakari Nuha, A. Abdolkarim, U. Aliyu, M.B. Gambo, M.A. Ibrahim, H. Abubakr, M.M. Bukar, H. Adama and P.T. Numan. 2011. Effects of onion (Allium cepa) and garlic (Allium sativum) on some Performance Charactristics of Broiler Chickens. Research Journal of Poultry Sciences, 4: 22-27. 
9. Chehrei, A., A. Nobakht and M.H. Shahir. 2011. The effects of different levels of biohebal feed supplement (contains thymus and garlic extracts) on performance, egg traits and blood biochemical and immunity parameters of laying hens. Iranian Journal of Veterinary Research and construction, 90: 58-65 (In Persian).

10. Chowdhury, S.R., S.D. Chowdhury and T.K. Smith. 2002. Effects of dietary garlic on cholesterol metabolism in laying hens. Poultry Science, 81: 1856-1862.

11. Courtis, J.A. and G.C. Wilson. 1990. Egg quality handbook. Queensland department of primary industries, Austeralia, pp: 23-90.

12. Demir, E., S. Sarica, M.A. Ozcan and M. Suicmez. 2003. The use nature feed additives as alternative for an antibiotic growth promoter in broiler diet. Poultry Science, 44: 44-45.

13. Essman, E.J. 1984. The medical uses of herbs. Fitoterapia, 55: 279-289.

14. Hertampf, J.W. 2001. Alternative antibacterial performance promoters. Poultry International, 40: 5052.

15. Hashemi Attar, M., H. Arshami, V. Esmaeilzadeh and R. Majidzadeh Heravi. 2010. Effect of different garlic levels on the performance and humeral immunity response in broilers. Iranian Journal of Animal Science Research, 2: 43-50 (In Persian).

16. Javandel, F., B. Navidshad, J. Seifdavati, Gh. Pourrahimi and S. Baniyaghoub. 2008. The favorite dosage of garlic meal as a feed additive in broiler chickens ratios. Pakistan Journal of Biological Sciences, 11: 1746-1749 (In Persian).

17. Khalid, Q., L. Sultan, M. Sawar and Y. Ahmad. 1995. Beneficial effects of Alium sativum linn in metabolism in laying hens. Poultry Science, 81: 1856-1862.

18. Kim, S.M., K. Kubota and A. Kobayashi. 1997. Anti oxidative activity of sulfur-containing flavor compounds in garlic. Bioscience Biotechnology and Biochemistry, 61: 1482-1485.

19. Konjufca, V.H., G.M. Pesti and R.I. Bakalli. 1997. Modulation of cholesterol levels of broiler meat by dietary garlic and copper. Poultry Science, 76: 1264-1271.

20. Kumar, M., R.S. Couhary and J.K. Vaishnav. 2005. Effect of supplemental prebiotic and turmerica in diet on the performance of broiler chicks during summer. Indian Journal of Poultry Science, 40: 137141.

21. Lee, K.W., H. Everts and A. Beyen. 2006. Dietary carvacrol lowers body weight gain but improves feed conversion in female broiler chickens. Journal of Applied Poultry Research, 12: 394-399.

22. Lewis, M.R., S.P. Rose, A.M. Mackenzie and L.A. Tucker. 2003. Effects of dietary inclusion of plant extracts on the growth performance of male broiler chickens. British Poultry Science, 44: 43-44.

23. National Research Council (NRC). 1994. Nutrient requirement of poultry. $9^{\text {th }}$ revised edition. National academy press. Washington, D.C.

24. Onibi, G.E., E. Oluwatoyin, A. Adebowale, N. Fajemisin and A.V. Adetunji. 2009. Response of and meat quality to garlic (Allium sativum) supplmention Afr. Jbroiler chickens in terms of performance. Agricultural Research, 4: 511-517.

25. Onyimonyi, A.E., P.C. Chukwuma and I. Chineenye. 2011. Growth and hypocholestrolemic properties of dry garlic powder on broiler. African Journal of Biotechnology, 11: 2666- 2671.

26. Peste, G.M. and B.R. Miller. 1994. User friendly feed formulation. (UFFDA). University of Georgia.

27. Pourabdollah, A. and A. Pourabdollah. 2001. Treating by Garlic and Onions. Nabi publication, Tehran. 256 pp.

28. Safamehr, A., Sh. Farajollah Zadeh, M.H. Shahiri and S. Khodaei. 2010. Effects of various levels of canola oil, grains, beans and garlic powder on the yield and qualitative traits of egg in laying hens. Veterinary Journal of Azad University, 4: 49-61 (In Persian).

29. Saffa, H.M. 2007. Effect of dietary garlic or fenugreek on cholosterol metabolism in lying hens. Egyptian Journal of Poultry Science, 27: 1207-1221.

30. SAS Institute. 2002. Statistical Analytical System User's Guide. Version 9.1. SAS Institute, Inc., Cary, NC.

31. Shen, R., J.D. Wang, P. Quanhai and L. Fang. 1999. Influence of Chinese medical herbadditive on lipid metabolism and productive performance of layers. Chinese Journal of Veterinary Science and Technology, 29: 31-32.

32. Unnikrishnan, M.C., K. Soudmini and R. Kuttan. 1990. Chemo protection of garlic extract toward cyclophoshamide toxicity in mice. Nutrition and Cancer, 13: 204-207.

33. Valizadeh, M. and M. Moghadam. 1994. Tentative plans in agriculture 1. Science pishtaze publication, pp: 75-100 (In Persian).

34. Wegmann, T. and O. Smithies. 1966. A Simple hemagglutination system requiring small amounts of red cells and antibodies. Transfusion, 6: 67-75. 


\title{
The Effects of Different Levels of Garlic and Fenugreek Powder on Performance, Egg Quality and Hematological Parameters and Antibody Titer of Commercial Laying Hens
}

\section{Seyed Mohammad Motamedi ${ }^{1}$ and Seyed Mozafar Mehdizade Taklimi ${ }^{2}$}

\author{
1- Ph.D. Student of Islamic Azad University Science and Research Branch of Tehran, Iran, Lecturer of Payam-e- \\ Noor University, Tehran, Iran. (Corresponding author: m_motamedi90@yahoo.com) \\ 2- Faculty Member of Research Institute of Animal Sciences, Karaj- Iran \\ Received: December 10, $2014 \quad$ Accepted: September 5, 2015
}

\begin{abstract}
This experiment was conducted to evaluate the effects of different levels of garlic and fenugreek powder on performance and immune response in laying hens. The aim of this experiment was to compare the effects of garlic and fenugreek powder on performance, egg quality and hematological parameters and antibody titer against Newcastle disease of commercial laying hens. The experiment was performed for 12 weeks (from 49 to 61 weeks of age) on 288 laying hens (Bovans strain), in a completely randomized design with 6 treatments, and 4 replicate for each treatment (12 birds/replicate). The results showed that garlic and fenugreek powder had no any effect on feed intake, egg mass, egg production percent, egg weight and feed conversion ratio $(p>0.05)$. The highest percentage of production was resulted by using $1 \%$ of garlic powder with $1 \%$ fenugreek powder. Dietary garlic and fenugreek powder had no significant effects on egg specific gravity, eggshell weight and eggshell thickness. The effect of garlic and fenugreek powder was significant on Haugh unit. Did not have any significant effects on hematological parameters and antibody titers against Newcastle disease of laying hens. The effect of garlic and fenugreek powder was significant in red blood cells. The lowest percentage of heterophile/lymphocyte, the highest percentage of lymphocyte were resulted by using $1 \%$ of garlic powder ( $p>0.05$ ). The overall results showed that using garlic and fenugreek powder until 1 percent improved the egg quality traits but it did not effect performance, blood parameters and immune response of laying hens.
\end{abstract}

Keywords: Garlic Powder, Fenugreek Powder, Antibody Titer, Performance, Blood Parameters and Laying Hen 